\title{
REVIEW
}

\section{Recent Application of Advanced MR Imaging to Predict Pseudoprogression in High-grade Glioma Patients}

\author{
Roh-Eul Yoo ${ }^{1,2}$ and Seung Hong $\mathrm{CHOI}^{1-4^{*}}$ \\ ${ }^{1}$ Department of Radiology, Seoul National University Hospital, \\ 101 Daehak-ro, Jongno-gu, Seoul 110-744, Republic of Korea \\ ${ }^{2}$ Department of Radiology, Seoul National University College of Medicine, \\ and Institute of Radiation Medicine, Seoul National University Medical Research Center \\ ${ }^{3}$ Center for Nanoparticle Research, Institute for Basic Science (IBS), Seoul National University \\ ${ }^{4}$ School of Chemical and Biological Engineering, Seoul National University
}

(Received May 8, 2015; Accepted October 28, 2015; published online December 28, 2015)

\begin{abstract}
Pseudoprogression is regarded as a subacute form of treatment-related change with a reported incidence of $20-30 \%$, occurring predominantly within the first three months after the completion of concurrent chemoradiotherapy (CCRT) in glioblastoma multiforme (GBM) patients. Occurrence of progressive lesions on conventional contrast-enhanced MR imaging may also accompany clinical deterioration, posing considerable diagnostic challenges to clinicians and radiologists. False interpretation of treatment-related change as true progression may lead to the cessation of effective first-line therapy (i.e., adjuvant temozolomide) and unnecessary surgery. Increasing awareness of the diagnostic challenge of the phenomenon has underscored the need for better imaging techniques that may aid in differentiating the treatment-related change from true progression. In this review, we discuss the recent applications of advanced MR imaging such as diffusion-weighted and perfusion-weighted imaging in the evaluation of treatment response in high-grade glioma patients and highlight their potential role in differentiating pseudoprogression from true progression.
\end{abstract}

\section{Keywords: diffusion-weighted imaging, perfusion-weighted imaging, pseudoprogression, high-grade glioma}

\section{Introduction}

Glioblastoma multiforme (GBM) is the most common primary malignancy in adults and is notorious for its intrinsic aggressiveness and dismal prognosis. ${ }^{1-3}$ Over the past decade, there has been a paradigm shift in the treatment of GBM due to a large-scale randomized controlled trial, which demonstrated clinically meaningful and significant survival benefits of the addition of temozolomide (TMZ) to radiotherapy for newly diagnosed GBM. ${ }^{4}$ Since then, TMZ has become a cornerstone of the treatment, and the current standard of care for GBM comprises maximal safe tumor resection

* Corresponding author, Phone: +82-2-2072-2584, Fax: +82-2-7477418, E-mail: verocay@snuh.org followed by radiation therapy with concurrent TMZ and adjuvant TMZ. ${ }^{4}$

With the paradigm shift in the GBM treatment, the occurrence of progressive enhancing lesions on magnetic resonance (MR) imaging immediately after completing concurrent chemoradiotherapy (CCRT) with TMZ has been increasingly recognized by radiologists and clinicians. The lesions tended to improve spontaneously without further treatment other than the adjuvant TMZ..$^{5-7}$ The term "pseudoprogression" was first coined in 2007 to describe the phenomenon mimicking tumor progression. ${ }^{7}$ Pseudoprogression is regarded as a subacute form of treatment-related change, which may accompany clinical deterioration. It is noteworthy that "pseudoprogression" and "radionecrosis" likely represent distinct entities, although the terms are 
occasionally used interchangeably. Pseudoprogression frequently occurs within 1-3 months after radiotherapy (RT) and often improves spontaneously, thus possibly implying higher treatment efficacy, whereas radionecrosis manifests in the later period (i.e., 6 months to several years after the end of CCRT) and often progresses with nonbeneficial complications. ${ }^{8,9}$

Pseudoprogression shows similar pathologic findings to those of radiation necrosis, such as edema, necrosis, and gliosis as well as endothelial thickening, hyalinization, thrombosis, and occlusion of vessels. ${ }^{1,6-8,10,11}$ Pseudoprogression may be attributed to early changes in the vascular endothelium and bloodbrain-barrier as well as oligodendroglial injury as an exaggerated response to effective therapy, which leads to inflammation and increased permeability. ${ }^{5}$ The abnormal enhancement seen in these patients may also be due to treatment-related cellular hypoxia, which triggers the expression of hypoxia-regulated molecules from the tumor and surrounding cells and the subsequent increase in vascular permeability. ${ }^{12}$

The reported incidence of pseudoprogression is $20-30 \%$, although there is a wide variation among previous reports. ${ }^{5-7}$ It has been established that pseudoprogression is strongly associated with the promoter methylation status of methylguanine methyltransferase (MGMT). The incidence of pseudoprogression was significantly higher in the methylated group than in the unmethylated group $(91 \%$ vs. $41 \%$, respectively; $P=0.0002) .{ }^{13}$

In parallel to the growing incidence of pseudoprogression following the incorporation of TMZ into the standard treatment, pseudoprogression has received increasing attention as a potential pitfall in the response evaluation. The occurrence of progressive lesions on conventional contrast-enhanced MR imaging may also accompany clinical deterioration, resulting in the false interpretation of the results as suggesting true progression. ${ }^{14}$ The false interpretation may lead to cessation of effective first-line therapy (i.e., adjuvant temozolomide) and unnecessary surgery. Due to the false interpretation, accurate determination of progression-free survival in clinical trials and eligibility for salvage treatment trials has been hindered..$^{15}$ Moreover, pseudoprogression has been shown to be associated with increased survival in some studies, which implies that pseudoprogression may reflect active inflammation against tumors. ${ }^{5}$

Although pathology is the reference standard in the differential diagnosis of pseudoprogression from true progression, it may still be challenging in certain cases, because pseudoprogression can contain some portions of viable tumor. Increasing awareness of the diagnostic challenge of the phenomenon has underscored the need for better imaging techniques that may aid in differentiating the treatment-related change from true progression. ${ }^{14}$ Among diverse advanced MR imaging techniques, diffusion-weighted imaging (DWI) and various perfusion-weighted imaging (PWI) techniques have long been considered attractive imaging biomarkers for treatment response in high-grade glioma patients. In this review, we discuss recent applications of advanced MR imaging in the evaluation of treatment response, and highlight their potential role in differentiating pseudoprogression from true progression.

\section{DWI}

DWI is an advanced imaging technique that utilizes a pair of strong diffusion gradients to detect microscopic water diffusion. Apparent diffusion coefficient (ADC) values from DWI are believed to reflect the cellularity in tumors. In recurrent tumors with high cellularity, diffusivity of water molecules is expected to be decreased due to relative reductions in extracellular space, as compared with treatment-related changes with low cellularity. Previous studies, although not strictly restricting the inclusion criteria to subacute radiation injury, have shown that there is a significant difference in ADC values between recurrent tumors and radiation injury. ${ }^{16-21}$ In the early study by Hein et al., both the mean ADC and ADC ratios were significantly lower in the recurrence group than the nonrecurrence group. ${ }^{18}$ However, another study by Asao et al. has found that the mean $A D C$ value was not shown to have a diagnostic value in the differential diagnosis. ${ }^{17}$ Discrepant results among the previous studies may be attributed to sampling bias, which is inevitable when regions of interest (ROIs) are manually selected within progressive lesions after CCRT with TMZ. It is speculated that the enhancing portion presumably represents a wide spectrum of histologic features comprising normal brain tissue, radiation necrosis, and highly cellular recurrent tumor and the results based on analysis of selected ROIs are likely to be prone to sampling bias, due to the cellular heterogeneity within the lesion. To eliminate the sampling bias, other studies have analyzed the ADC of the entire volume of the enhancing portion instead of manually selecting ROIs. The collective data from the whole enhancing tumor enables in-depth histogram analysis, which better reflects the cellular heterogeneity (different tissue diffusion characteristics) by taking into account all the tissue components of the enhancing portion that contribute to group differences between true progression and pseudoprogression. Various parameters from ADC histograms, such as the mean, kurtosis, skewness, and the fifth percentile of cumulative ADC histograms (the point at which $5 \%$ of the voxel values that form the cumulative ADC histogram are found to the left), have been previously investigated. ${ }^{19,22,23}$ The 
mean ADC value has been found to have the discriminative ability to identify true progression or pseudoprogression in some studies ${ }^{19}$ but not in others, ${ }^{22,23}$ while the fifth percentile has been consistently shown to be helpful in distinguishing the two..$^{22,23}$ Song et al. reported that true progression could be differentiated from pseudoprogression with a sensitivity of $90 \%$ and a specificity of $90 \%$ using a cutoff fifth percentile value of $892 \times 10^{-6} \mathrm{~mm}^{2} / \mathrm{sec}$ (Figs. 1, 2) ${ }^{23}$ Another study by Chu et al. has shown that the fifth percentiles of cumulative ADC histograms at both standard $(b=1000 \mathrm{sec} /$ $\left.\mathrm{mm}^{2}\right)$ and high $\left(\mathrm{b}=3000 \mathrm{sec} / \mathrm{mm}^{2}\right) \mathrm{b}$ values were significantly lower in the true progression group than in the pseudoprogression group $(P=0.049$ and $P<0.001$, respectively) (Figs. 3, 4). ${ }^{22}$ Diagnostic performances of the fifth percentiles at the $b$ values are provided in Table 1. The results suggested that the fifth percentile of the cumulative ADC histogram obtained at the high $\mathrm{b}$ value could be the most promising parameter in distinguishing the two groups. Meanwhile, the mean ADC values at both the standard and high $b$ values were not significantly different between the two groups. The discordant results of the mean ADC value and the fifth percentiles of the cumulative ADC histograms may be explained by the finding that true progression consists of more cellular components, as reflected by a higher relative frequency at low ADC values, than does pseudoprogression, even though both true progression and pseudoprogression comprise variable portions of viable tumors and post-CCRT necrosis. ${ }^{24,25}$ With regard to other histogram parameters such as kurtosis and skewness, no significant difference was found between the two groups. ${ }^{19}$

\section{Perfusion-weighted Imaging}

\section{Dynamic susceptibility-weighted contrast-enhanced perfusion MR imaging}

Neoplasm is known to contrast with a necrotic region in that it mainly shows neoangiogenesis, as defined by an irregular meshwork of newly formed vessels arising from coexisting vessels, while the latter reveals extensive vascular injury and tissue ischemia with vascular endothelial damage, hyalinization of vessels, thrombosis, and increased permeability. ${ }^{26} \mathrm{~A}$ combination of neocapillary formation and dilation of preexisting vasculature, in turn, is thought to cause increase in the cerebral blood volume (CBV) in tumor progression. ${ }^{27-29}$

Over the past few years, the diagnostic value of CBV from dynamic susceptibility-weighted contrast-enhanced perfusion MR imaging (DSC-PWI) in differentiating pseudoprogression from true progression has been investigated in several studies. ${ }^{9,14,24,30-34}$ Kong et al. found a significant difference in the maximum rCBV ratio between true progression and pseudoprogression $(P=$ $0.003) .{ }^{24}$ In a subgroup analysis according to the MGMT methylation status, a significant difference of the rCBV between the two groups was found in the unmethylated group $(P=0.009)$ but not in the methylated group $(P=$ $0.258)$. Given these findings, the authors suggested first evaluating the methylation status of the MGMT promoter gene when attempting to differentiate the two groups. In cases with methylation, they recommended considering pseudoprogression first, on the basis of the high incidence of pseudoprogression in the group and continuing TMZ until the subsequent follow-up imaging. ${ }^{13,24}$ On the other hand, as for the unmethylated group, in which rCBV could have higher predictability of true progression, the authors suggested that the possibility of true progression should be considered in those with an $\mathrm{rCBV}$ greater than 1.47 and that the second-line treatment needs to be started.

In another study by Boxerman et al., which investigated the value of longitudinal trends in $\mathrm{rCBV}$ for predicting pseudoprogression following CCRT, only the change in $\mathrm{rCBV}$ at the first subsequent follow-up $(-0.84$ in pseudoprogression vs. 0.84 in true progression; $P=0.001)$ and the overall linear trend in $\mathrm{rCBV}$ after initial progressive enhancement (negative in pseudoprogression vs. positive slope in true progression; $P$ $=0.04$ ), but not the mean rCBV at initial progressive enhancement, were found to be significantly different between true progression and pseudoprogression. ${ }^{31}$ Based on the results, the authors suggested that longitudinal trends in rCBV may be more useful than absolute rCBV values in distinguishing pseudoprogression from true progression. The results are in keeping with those of the previous study by Mangla et al., in which nine patients with suspected pseudoprogression had a mean decrease in rCBV of $41 \%$ at 1 month after CCRT, while 12 patients with suspected true progression had a mean increase in $\mathrm{rCBV}$ of $12 \% .^{14}$

Meanwhile, Tsien et al. hypothesized that the parametric response map (voxel-wise method of image analysis) of rCBV would better predict clinical outcomes, as compared with standard imaging methods including percent change of rCBV and relative cerebral blood flow, given the cellular heterogeneity within tumors. ${ }^{34}$ Paradoxically, the fractional tumor volume with a decrease in rCBV was found to be significantly higher in true progression than in pseudoprogression $(P<0.01)$. Average percent changes of rCBV or relative cerebral blood flow, on the other hand, did not significantly differ between the two groups. However, the results should be interpreted with caution because the incidence of treatment-related change such as pseudoprogression could have been exaggerated by the use of a relatively higher total radiation dose. In addition, it 

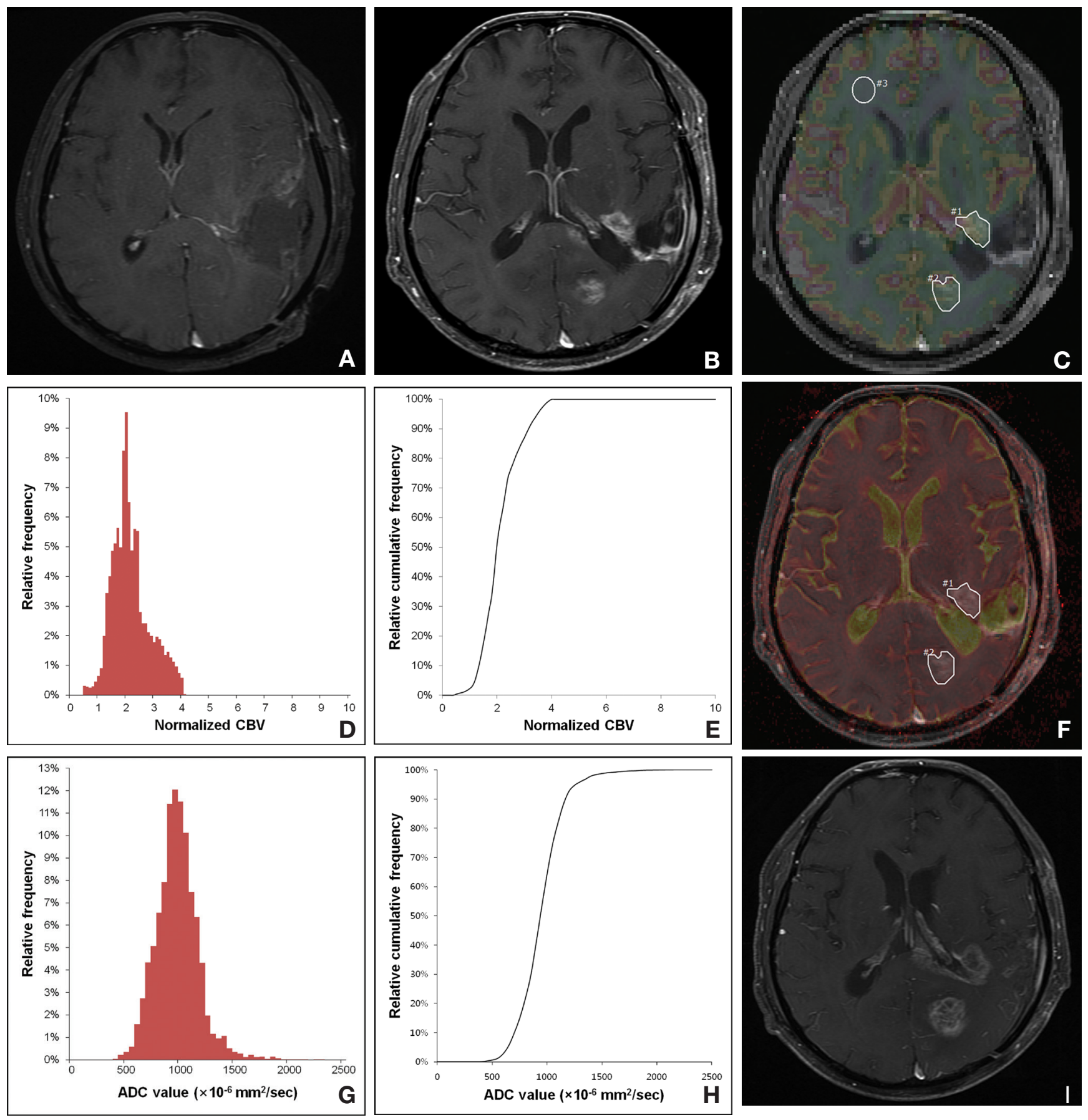

Fig. 1. MR images, nCBV histograms, and ADC histograms in a 59-year-old man with glioblastoma. (A) Axial CE $\mathrm{T}_{1} \mathrm{~W}$ image taken immediately after gross total resection shows no definite enhancing lesion. (B) Three weeks after CCRT with TMZ, new enhancing lesions are visible in left periventricular white matter and in left occipital lobe. (C) nCBV map, which is displayed as color overlay on $\mathrm{CE} \mathrm{T}_{1} \mathrm{~W}$ image taken 3 weeks after CCRT with TMZ, shows slightly increased nCBV in lesion (polygonal ROIs \#1 and \#2) compared with contralateral white matter (round ROI \#3). (D) Normalized CBV histograms and (E) cumulative histograms of enhancing lesions. (F) ADC map, which is displayed as color overlay (in hot scale) on $\mathrm{CE} \mathrm{T}_{1} \mathrm{~W}$ image, shows slightly decreased ADC value for lesion (polygonal ROIs \#1 and \#2). (G) ADC histograms and (H) cumulative histograms of enhancing lesion. (I) After continuing TMZ for 1 month, patient visited emergency room due to involuntary movement. On second follow-up MR imaging that was performed during visit to emergency room, there was increase in enhancement of lesions without further treatment. After 3 months, patient passed away in spite of the continuation of adjuvant TMZ, which is compatible with true progression. ADC, apparent diffusion coefficient; CBV, cerebral blood volume; CCRT, concurrent chemoradiotherapy; CE, contrast-enhanced; nCBV, normalized CBV; ROIs, regions of interest; $\mathrm{TMZ}$, temozolomide; $\mathrm{T}_{1} \mathrm{~W}, \mathrm{~T}_{1}$-weighted. (Reprinted, with permission, from reference [23].) 

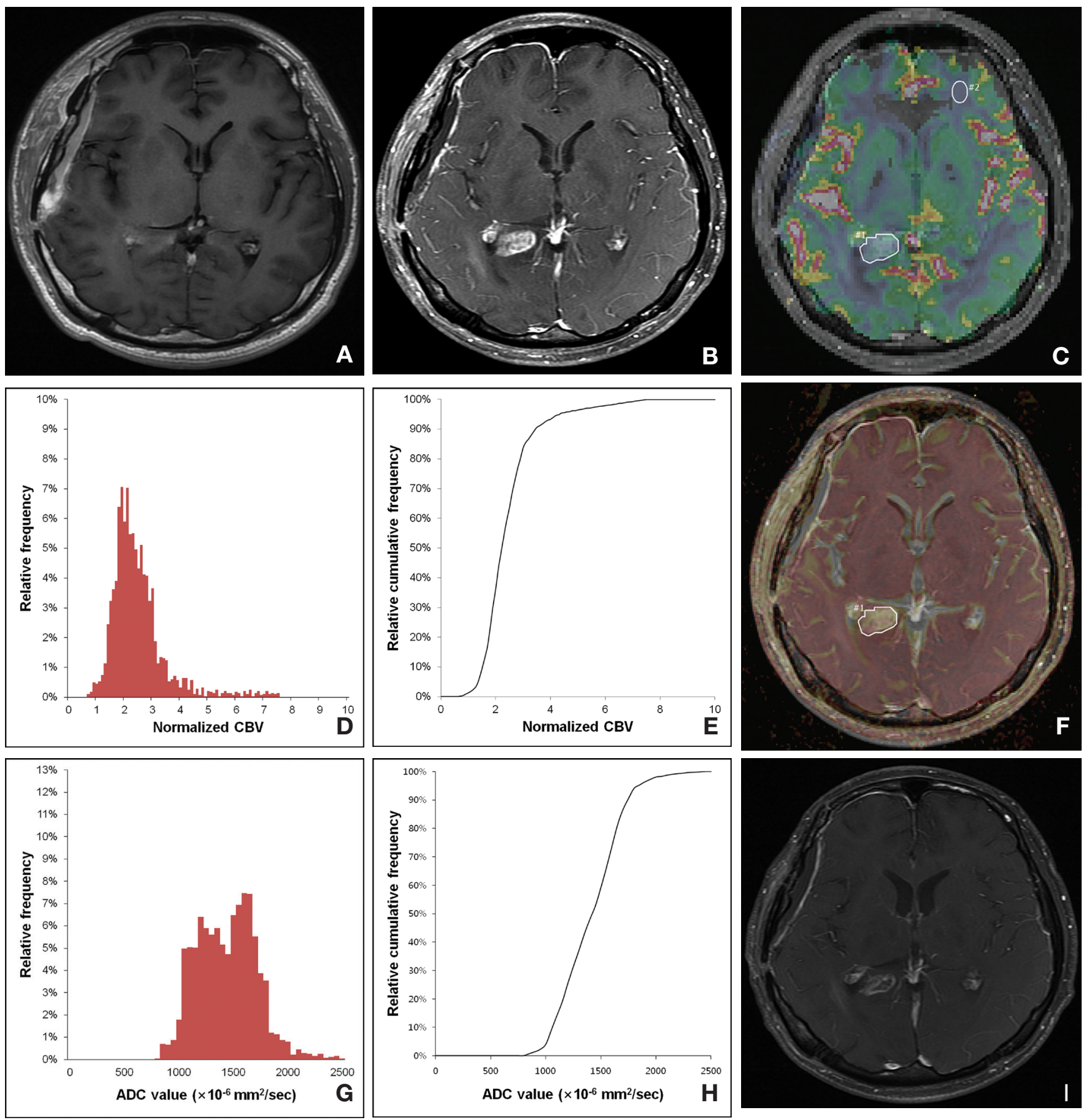

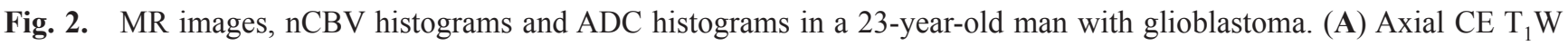
image taken immediately after gross total resection shows subtle enhancing lesion at the right splenium of corpus callosum and (B) enlargement of enhancing lesion, 1 month after CCRT with TMZ. (C) nCBV map, which is displayed as color overlay on $\mathrm{CE} \mathrm{T}_{1} \mathrm{~W}$ image taken 1 month after CCRT with TMZ, shows slightly increased nCBV in lesion (polygonal ROI \#1) compared with contralateral white matter (round ROI \#2). (D) Normalized CBV histograms and (E) cumulative histograms of enhancing lesion. (F) ADC map, which is displayed as color overlay (in hot scale) on $\mathrm{CE} \mathrm{T}_{1} \mathrm{~W}$ image, shows increased ADC value for lesion (polygonal ROI \#1). (G) ADC histograms and (H) cumulative histograms of enhancing lesion. (I) On second follow-up MR imaging that was performed during outpatient visit after continuing TMZ for 3 months, enhancement of lesion was decreased without further treatment, which confirms pseudoprogression. ADC, apparent diffusion coefficient; CBV, cerebral blood volume; CCRT, concurrent chemoradiotherapy; CE, contrast-enhanced; nCBV, normalized CBV; ROI, region of interest; TMZ, temozolomide; $\mathrm{T}_{1} \mathrm{~W}, \mathrm{~T}_{1}$-weighted. (Reprinted, with permission, from reference [23].) 

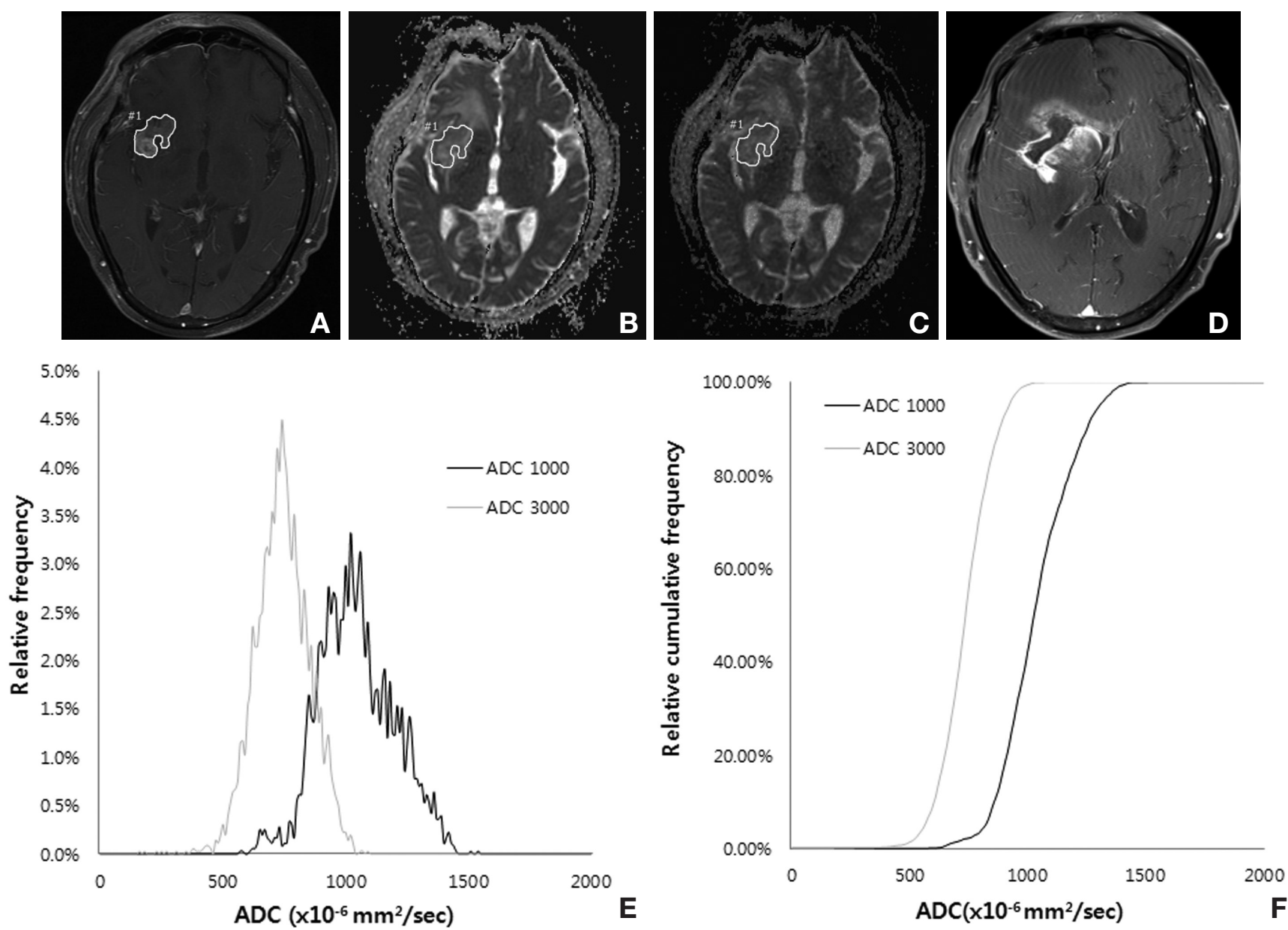

Fig. 3. True progression in a 56-year old man with glioblastoma. (A) Axial contrast-enhanced $\mathrm{T}_{1}$-weighted MR image obtained within 1 month after surgical resection and the end of CCRT shows a newly developed enhancing lesion in the right insular cortex. Axial (B) $\mathrm{ADC}_{1000}$ and $(\mathbf{C}) \mathrm{ADC}_{3000}$ maps with regions of interest corresponding to (A) show the decreased ADC value of the lesion. (D) After a 3-month continuation of TMZ, lesion enhancement is shown with an internal necrotic cystic component, which is suggestive of true progression. Corresponding (E) $\mathrm{ADC}_{1000}$ and $\mathrm{ADC}_{3000}$ and $(\mathbf{F})$ cumulative histograms. The ADCs decreased when the b value was increased from 1000 to $3000 \mathrm{sec} / \mathrm{mm}^{2}$ and at a relatively higher frequency at the low ADC value. ADC, apparent diffusion coefficient; CCRT, concurrent chemoradiotherapy; TMZ, temozolomide. (Reprinted, with permission, from reference [22].)

should be noted that their analysis was based on early perfusion data acquired at week 3 during CCRT, which is not a standard time for follow-up MR imaging.

As with ADC, histogram analyses of perfusion data have also been undertaken in a few studies. Song et al. analyzed predictive values of the $70^{\text {th }}, 90^{\text {th }}$, and $95^{\text {th }}$ percentile points from cumulative $\mathrm{nCBV}$ (relative CBV of the tumor normalized with respect to the contralateral normal white matter) histograms as well as the mean and peak height from nCBV histograms. ${ }^{23}$ In contrast to parameters from ADC histograms, none of the parameters from nCBV histograms were shown to be discriminatory predictors of pseudoprogression (Figs. 1, 2). Other histogram parameters including histogram shape (kurtosis) and asymmetry (skewness) have also been investigated. Baek et al. hypothesized that changes in kurtosis and skewness derived from $n C B V$, between the first and second post-CCRT fol- low-up, may serve as an imaging biomarker for the change in heterogeneous tumor vascularity of highgrade gliomas and ultimately as a predictor for early treatment response to CCRT. The percent change of skewness and kurtosis were classified into four histographic patterns: category 1 with positive skewness and leptokurtosis, category 2 with positive skewness and platykurtosis, category 3 with negative skewness and leptokurtosis, and category 4 with negative skewness and platykurtosis. Among various histogram parameters (maximum, mode, range, percent change of skewness and kurtosis, and histographic patterns), which showed statistical significance in univariate analyses, only the maximum and histographic pattern were found to be significant independent predictors of early tumor progression. Overall, the histographic pattern was the best independent predictor with an odds ratio of 3.51 . 

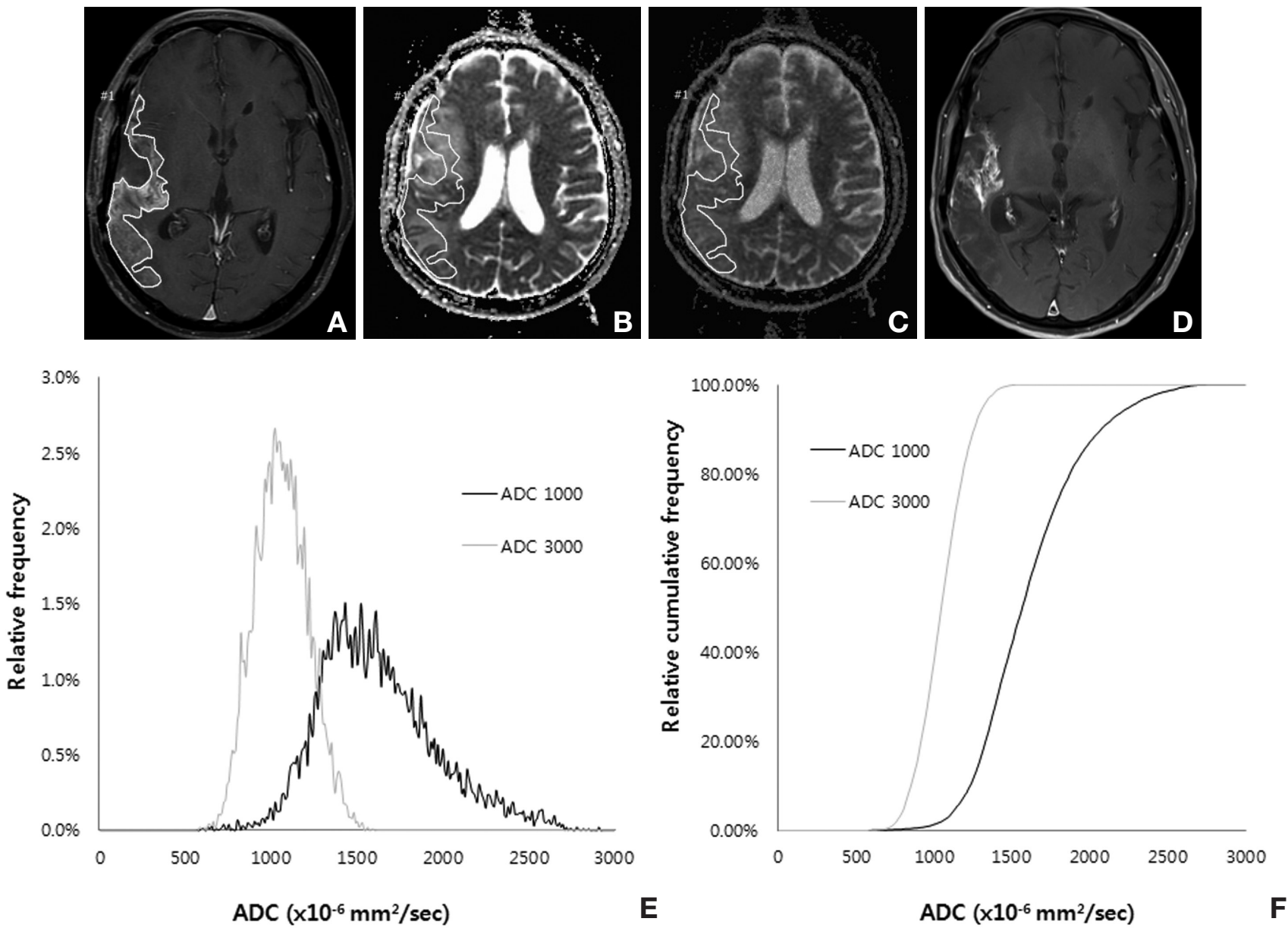

Fig. 4. Pseudoprogression in a 58-year old man with glioblastoma. (A) Axial contrast-enhanced $\mathrm{T}_{1}$-weighted MR image obtained within 1 month after surgical resection and the end of CCRT shows a newly developed enhancing lesion in the right frontotemporal lobe. Axial (B) $\mathrm{ADC}_{1000}$ and $(\mathbf{C}) \mathrm{ADC}_{3000}$ maps with regions of interest corresponding to (A) show the decreased ADC of the lesion. (D) After a 6-month continuation of TMZ, lesion enhancement decreased without further treatment, which was suggestive of pseudoprogression. Corresponding (E) $\mathrm{ADC}_{1000}$ and $\mathrm{ADC}_{3000}$ and (F) cumulative histograms. The ADCs decreased when the $\mathrm{b}$ value was increased from 1000 to $3000 \mathrm{sec} / \mathrm{mm}^{2}$ and dispersed over a wider range. ADC, apparent diffusion coefficient; CCRT, concurrent chemoradiotherapy; TMZ, temozolomide. (Reprinted, with permission, from reference [22].)

In addition to histogram parameters (rCBV mean, mode, maximum, and histogram width), Hu et al. studied the correlation between a voxel-based thresholding metric, termed pMRI fractional tumor burden (pMRIFTB), and histologic tumor fraction or overall survival.

The pMRI-FTB demonstrated the strongest association with the histologic tumor fraction $(\mathrm{r}=0.82 ; P$ $<0.0001)$ and was the only parameter that correlated with overall survival $(P<0.02) .^{33}$

Predictive values of other DSC-PWI parameters such as relative peak height (rPH) and percent signal change (PSR) have also been studied in some previous reports. The rPH and PSR are calculated using the following formulas:

$$
\begin{gathered}
\mathrm{rPH}=\frac{\left(\mathrm{S}_{0}-\mathrm{S}_{\text {min }}\right)_{\text {lesion }}}{\left(\mathrm{S}_{0}-\mathrm{S}_{\text {min }}\right)_{\text {contralateral normal-appearing white matter }}} \\
\mathrm{PSR}=\left(\mathrm{S}_{1}-\mathrm{S}_{\text {min }}\right) /\left(\mathrm{S}_{0}-\mathrm{S}_{\text {min }}\right)
\end{gathered}
$$

where $\mathrm{S}_{0}$ is the baseline signal intensity prior to contrast injection, $S_{\min }$ is the minimal signal intensity at peak of contrast bolus, and $\mathrm{S}_{1}$ is the end signal intensity at $60 \mathrm{sec}$. The $\mathrm{rPH}$ is defined as the maximal change in signal intensity and is known to correlate with rCBV. ${ }^{35}$ PSR is influenced by the size of the extravascular space and contrast leakage; therefore, lower PSR implies delayed return of the perfusion curve to baseline and thus higher vascular permeability. ${ }^{35,36}$ Young et al. reported that pseudoprogression had significantly lower median $\operatorname{rCBV}(P=0.009)$ and $\mathrm{rPH}(P<0.001)$ and higher PSR $(P=0.039)$ than true progression. This observation is thought to mirror the reduced or absent neoangiogenesis as well as low-grade leakiness attributable to inflammation and edema in pseudoprogression. ${ }^{9}$

Accurate assessment of rCBV in DSC-PWI necessitates intravascular localization of contrast agents. Patients 
Table 1. Summary of diagnostic performances of advanced MR imaging parameters

\begin{tabular}{|c|c|c|c|c|c|c|}
\hline \multirow{2}{*}{$\begin{array}{l}\text { Imaging } \\
\text { technique }\end{array}$} & \multirow[t]{2}{*}{ Study/Year } & \multirow[t]{2}{*}{ Parameter } & \multirow[t]{2}{*}{ Cutoff } & \multicolumn{3}{|c|}{ Diagnostic performance } \\
\hline & & & & Sensitivity (\%) & Specificity (\%) & AUC \\
\hline \multirow[t]{4}{*}{ DWI } & Lee et al./2012 & Mean ADC & $1200 \times 10^{-6} \mathrm{~mm}^{2} / \mathrm{sec}$ & $80(8 / 10)$ & $83.3(10 / 12)$ & NA \\
\hline & Song et al./2013* & $\begin{array}{l}\text { Fifth percentile } \\
\text { at the standard } \\
\text { b value }\end{array}$ & $892 \times 10^{-6} \mathrm{~mm}^{2} / \mathrm{sec}$ & $90(9 / 10)$ & $90(9 / 10)$ & $\begin{array}{c}0.880 \\
{[0.658-0.981]}\end{array}$ \\
\hline & Chu et al./2013* & $\begin{array}{l}\text { Fifth percentile } \\
\text { at the standard } \\
\text { b value }\end{array}$ & $929 \times 10^{-6} \mathrm{~mm}^{2} / \mathrm{sec}$ & $73(11 / 15)$ & $73(11 / 15)$ & $\begin{array}{c}0.757 \\
{[0.563,0.896]}\end{array}$ \\
\hline & & $\begin{array}{l}\text { Fifth percentile } \\
\text { at the high } b \\
\text { value }\end{array}$ & $645 \times 10^{-6} \mathrm{~mm}^{2} / \mathrm{sec}$ & $93(14 / 15)$ & $100(15 / 15)$ & $\begin{array}{c}0.981 \\
{[0.847,1.000]}\end{array}$ \\
\hline \multirow[t]{3}{*}{ DSC-PWI } & Mangla et al./2010 & $\begin{array}{l}\text { Percentage } \\
\text { change } \\
\text { in rCBV }\end{array}$ & $0 \%$ & $77(9 / 12)$ & $86(6 / 7)$ & $\begin{array}{c}0.85 \\
{[\mathrm{NA}]}\end{array}$ \\
\hline & Kong et al./2011 & $\begin{array}{l}\text { Maximum } \\
\text { rCBV }\end{array}$ & 1.47 & $82(27 / 33)$ & $78(20 / 26)$ & NA \\
\hline & Baek et al./2012 & $\begin{array}{l}\text { Histographic } \\
\text { pattern }\end{array}$ & Category 3 & $86(36 / 42)$ & $89(33 / 37)$ & $\begin{array}{c}0.934 \\
{[0.855,0.977]}\end{array}$ \\
\hline \multirow[t]{2}{*}{ DCE-MRI } & Suh et al./2013 & mAUCRH & 0.31 & $90(38 / 42)$ & $83(31 / 37)$ & $\begin{array}{c}0.901 \\
{[0.791-0.976]}\end{array}$ \\
\hline & Yun et al./2015 & Mean $\mathrm{K}_{\text {trans }}$ & $0.347 \mathrm{~min}^{-1}$ & $59(10 / 17)$ & $94(15 / 16)$ & $\begin{array}{c}0.756 \\
{[0.575,0.888]}\end{array}$ \\
\hline
\end{tabular}

Numbers in parentheses are raw data. Numbers in square brackets indicate $95 \%$ confidence intervals. $*$ The standard and high $\mathrm{b}$ values are $1000 \mathrm{sec} / \mathrm{mm}^{2}$ and $3000 \mathrm{sec} / \mathrm{mm}^{2}$, respectively. ADC, apparent diffusion coefficient; AUC, area under the receiver-operating-characteristic curve; DCE-MRI, dynamic contrast-enhanced magnetic resonance imaging; DSCPWI, dynamic susceptibility-weighted contrast-enhanced perfusion MR imaging; mAUCRH, mean area under the time signal-intensity curves ratio at a higher curve; NA, not available; rCBV, relative cerebral blood volume.

with malignant brain tumors are prone to the blood-brain barrier disruption, particularly following CCRT, which may in turn result in the underestimation of $\mathrm{rCBV}$, due to rapid extravasation of low-molecular-weight gadolinium-based contrast agents. ${ }^{12,29,37,38}$ In this context, ferumoxytol (AMAG Pharmaceuticals, Cambridge, Massachusetts, USA) a very small superparamagnetic iron oxide nanoparticle with a relatively large iron particle (approximately $30 \mathrm{~nm}$ ), may be an attractive alternative to gadolinium-based contrast agents because of its potential to act as a blood pool agent shortly after administration. ${ }^{39}$ Recently, Gahramanov et al. measured rCBV values using both gadoteridol (ProHance, Bracco Diagnostic, Princeton, New Jersey, USA) and ferumoxytol in GBM patients with progressive enhancement after CCRT and correlated them with survival. ${ }^{32}$ In cases with ferumoxytol, rCBV correlated well with overall survival with low rCBV $(\leq 1.75)$, resulting in significantly improved survival (hazard ratio, 0.098; $P=0.004$ ). Conversely, as for gadoteridol, a significant association between $\mathrm{rCBV}$ and survival was observed only after leakage correction. The results suggest that ferumoxytol may provide a reliable and simplified means for rCBV assessment with no need for leakage correction and aid in the diagnosis of pseudoprogression and the prediction of survival.

The diagnostic performances of the parameters from DSC-PWI are summarized in Table 1.

\section{Dynamic contrast-enhanced $T_{1}$-weighted perfusion MR imaging}

Quantitative pharmacokinetic parameters derived from dynamic contrast-enhanced $\mathrm{T}_{1}$-weighted perfusion $\mathrm{MR}$ imaging have been thought to reflect microcirculatory structure and function in tumors. $\mathrm{K}_{\text {trans }}$ is defined as the volume transfer constant between the plasma and extravascular extracellular space and $\mathrm{v}_{\mathrm{e}}$, also known as the leakage space, represents the extravascular extracellular space (EES) per unit volume of tissue. Both $\mathrm{K}_{\text {trans }}$ and $\mathrm{v}_{\mathrm{e}}$ are well-known imaging biomarkers for permeability. ${ }^{40,41}$ In addition, $\mathrm{v}_{\mathrm{p}}$ (blood plasma volume per unit volume of tissue) may reflect angiogenic activity in tumors. ${ }^{42}$

The various parameters have been exploited to gain insights into tumor neovasculature, which is characterized by neoangiogenesis and increased vascular permeability. In a recent study by Yun et al., the mean $\mathrm{K}_{\text {trans }}$ and $\mathrm{v}_{\mathrm{e}}$ but not the mean $\mathrm{v}_{\mathrm{p}}$ were found to 
A CE T1WI
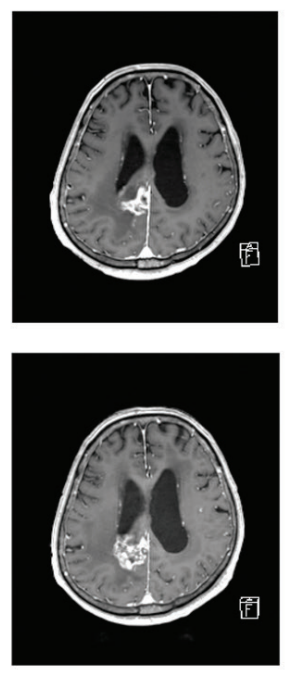

B
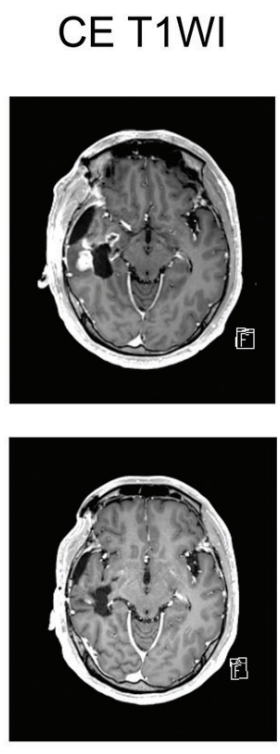

Ktrans
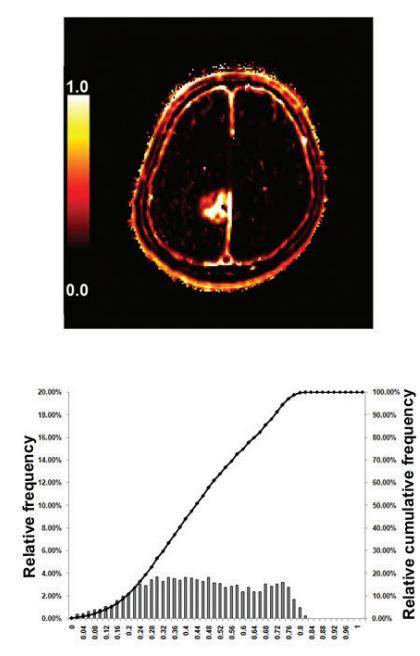

Ktrans $\left(\min ^{-1}\right)$

Ktrans
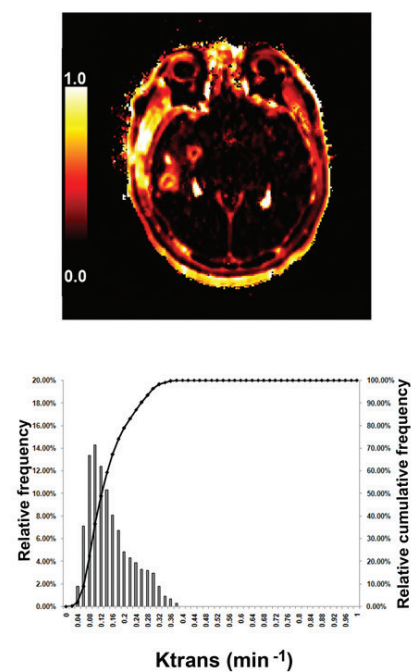

Ve
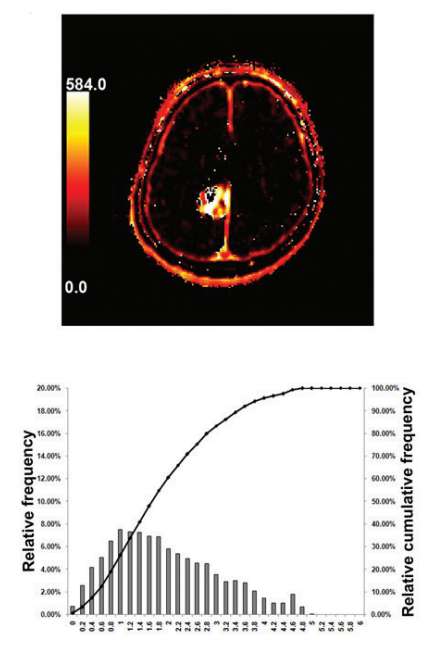

Ve

Ve
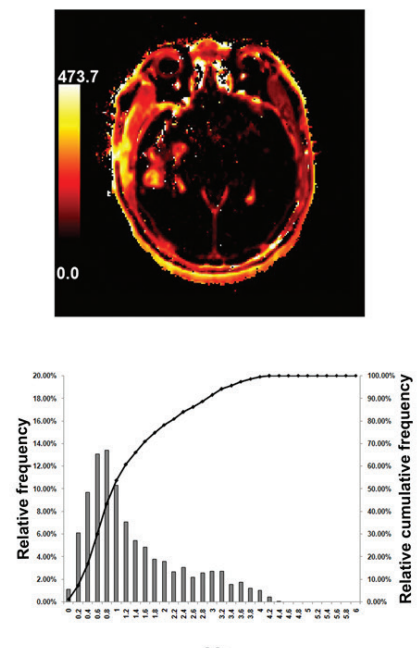

$V p$
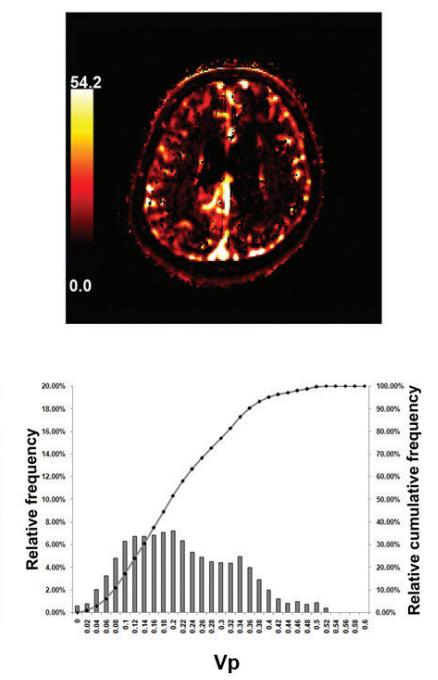

$V p$
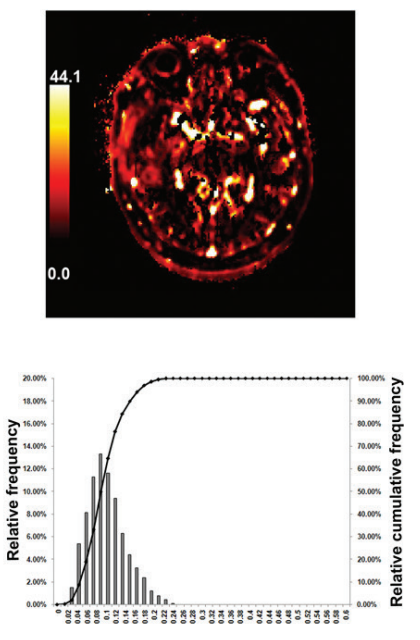

$\mathrm{Vp}$

Fig. 5. Representative dynamic contrast-enhanced MR imaging-derived pharmacokinetic parameter maps and histograms in true progression and pseudoprogression. (A) Images in a 79-year-old patient with GBM and true progression. The maps for the pharmacokinetic parameters in the enhancing area show increased $\mathrm{K}_{\text {trans }}, \mathrm{v}_{\mathrm{e}}$, and $\mathrm{v}_{\mathrm{p}}$ values within the enhancing portion compared with the corresponding maps in (B). According to findings at follow-up MR imaging after six cycles of adjuvant TMZ (top right MR image), the patient was confirmed as having true progression. (B) Images in a 43-year-old patient with GBM and pseudoprogression. Histograms for pharmacokinetic parameters in the enhancing area show the left shift of the lines representing relative cumulative frequencies for $\mathrm{K}_{\text {trans }}, \mathrm{v}_{\mathrm{e}}$, and $\mathrm{v}_{\mathrm{p}}$ compared with the corresponding histograms in (A). This shift means that the values in true progression are mainly distributed within the higher values, as compared with those in pseudoprogression. According to findings at follow-up MR imaging after six cycles of adjuvant TMZ (top right MR image), the patient had pseudoprogression. CE, contrast enhanced; GBM, glioblastoma multiforme; MR, magnetic resonance; $\mathrm{T}_{1} \mathrm{WI}$, $\mathrm{T}_{1}$-weighted imaging. (Reprinted, with permission, from reference [43].)

be significantly higher in true progression than in pseudoprogression (Fig. 5). ${ }^{43}$ The mean $\mathrm{K}_{\text {trans }}$ turned out to be the only independent predictor for true progression. Although the quantitative model-based pharmacokinetic parameters may have the potential to measure true physiologic mechanisms, their clinical utility is limited by several factors, including parameter coupling, water exchange, arterial input function measurement, and model fit instability. ${ }^{44}$ In contrast, semi-quantitative model-free parameters such as initial area under the time signal-intensity curve (IAUC) do not require measurement of an arterial input function and are less susceptible to fit failures on noisy data and thus have been suggested to be more reproducible measures. ${ }^{45}$ Nonetheless, the major drawback of the semiquantitative parameters is their lack of clear biologic association. To overcome the drawback, Suh et al. proposed the use of a ratio of initial and final area under the 
time signal-intensity curves (AUCRs) to discriminate between true progression and pseudoprogression, under the hypothesis that the IAUC represents the degree of early contrast leakage into EES, while the final area under the time signal-intensity curve (FAUC) reflects the amount of contrast leakage within the EES. ${ }^{46}$ In their study, a two-component mixture normal distribution was used for better fitting of histogram curves. ${ }^{47}$ The mean area under the time signal-intensity curves ratio at a higher curve (mAUCRH), three cumulative histogram parameters (AUCR50, AUCR75, and AUCR90), and the area under the time signal-intensity curves ratio at mode (AUCRmode) were all shown to be significantly higher in true progression than in pseudoprogression. It has been speculated that a relatively high IAUC30 in true progression may be explained by hypervascularity and neoangiogenesis of the viable tumor, while a relatively high FAUC30 in pseudoprogression may be attributable to lower tumor cellularity and greater tissue damage, which in turn causes greater retention of the contrast agent in the EES. Furthermore, mAUCRH was determined to be the best single predictor of pseudoprogression.

The diagnostic performances of the parameters from dynamic contrast-enhanced $\mathrm{T}_{1}$-weighted perfusion $\mathrm{MR}$ imaging are summarized in Table 1.

\section{Arterial spin-labeling perfusion MRI}

Although DSC-PWI remains the mainstay of perfusion MR imaging for the evaluation of tumor vasculature, there has been growing interest in arterial spin-labeling perfusion MRI (ASL-PWI) over the past few years, as it allows for consecutive and absolute quantification of tissue perfusion with no need for contrast administration. ${ }^{48,49}$ ASL-PWI would be of particular benefit to pediatric patients or those with poor renal function, who are at risk for developing nephrogenic systemic fibrosis.

The clinical utility of ASL-PWI in brain tumors has been investigated in several studies. ${ }^{48,50-54}$ More recently, Choi et al. explored whether ASL-PWI has an added value in distinguishing pseudoprogression from true progression via qualitative and semi-quantitative analyses. ${ }^{55}$ In qualitative analysis, diagnostic accuracy was increased from $75.8 \%$ to $88.7 \%$ after the addition of ASL-PWI, although the improvement did not reach statistical significance. Regarding the semi-quantitative analysis, the incidence of ASL grade III (i.e., tumor perfusion comparable to that of blood vessels) was significantly higher in true progression than in pseudoprogression. In addition, the maximum and range of DSC perfusion histogram parameters differed significantly according to ASL grade. Subsequent analysis showed ASL grade to be an independent predictor of early tumor progression (odds ratio, 4.73; $P=0.0017$ ), with an AUC of 0.774 , a sensitivity of $79.4 \%$, and a specificity of $64.3 \%$. However, despite the advantage that ASL-PWI is less likely to be influenced by bloodbrain-barrier breakdown and susceptibility artifacts, it has a lower spatial resolution and a longer scan time, as compared with DSC-PWI, which may reduce its clinical utility. ${ }^{53}$

\section{Multiparametric Imaging}

Acknowledging that DWI and PWI represent different aspects of tumor biology, efforts have been made to increase the accuracy for the differential diagnosis of pseudoprogression from true progression by simultaneous use of the multiple parameters. In a study by Cha et al., subtracted histograms were made from combined histograms (rCBV $\times$ ADC) of initial and follow-up MR imaging. ${ }^{56}$ Diagnostic performance (AUC) of the subtracted histogram mode with a multiparametric approach was higher than that of a uniparametric approach $(0.877$ vs. 0.801 , respectively), with a sensitivity of $81.8 \%$ and a specificity of $100 \%$. Furthermore, a high mode of rCBV on the subtracted histogram was found to be the best predictor of true progression $(P<0.001)$ and worse survival $(P<0.003)$. More recently, Park et al. demonstrated the added value of volume-weighted voxel-based multiparametric clustering (VVMC) over single parametric methods, namely ADC, nCBV, and IAUC. ${ }^{57}$ k-means clustering, a data processing and image segmentation method, was used to group voxel-based data points into three clusters with varying proportions of viable tumor and treatment-related changes (i.e., predominant viable tumor, mixed viable tumor and treatment-related change, and predominant treatment-related change). A total cluster score was calculated by summing volume-weighted cluster scores, which are determined by multiplying cluster scores (sum of parameter scores for nCBV, ADC, and IAUC) by cluster volume fractions. VVMC was shown to be more accurate and reproducible than the single parametric methods for discriminating pseudoprogression from true progression, with the sensitivity, specificity, and accuracy of $87-91.1 \%, 87.1-91.1 \%$, and $87.0-90.9 \%$ at a cutoff value of 5.91 or 5.94, respectively. Although the diagnostic performance of the multiparametric approach tends to be higher than that of the single-parameter analyses, further studies on the discriminatory value of other combinations of various parameters would be warranted to improve the diagnostic performance.

\section{Conclusion}

Although pathology is the reference standard in the differential diagnosis of pseudoprogression from true progression, it may still be challenging in certain cases, because pseudoprogression can contain some portions of viable tumor. ${ }^{24}$ Therefore, multidisciplinary team 
approaches taking into account clinical, radiologic, and pathologic findings are mandatory to increase the diagnostic accuracy of pseudoprogression. The low fifth percentile of the cumulative ADC histograms from DWI reflects hypercellularity in viable tumors, while various parameters from $\mathrm{PWI}$ - high $\mathrm{rCBV}, \mathrm{rPH}, \mathrm{K}_{\text {trans }}$, $\mathrm{V}_{\mathrm{e}}$, IAUC, AUCR, and low PSR - reflect neoangiogenesis and increased vascular permeability in true progression. It is noteworthy that the specific cutoff values, as reported by many previous studies, may need to be adjusted for applications in different clinical settings because of the difference in image acquisition techniques and analysis methods. Nonetheless, additional diagnostic clues provided by the diverse advanced MR techniques, which represent different aspects of tumor biology, may aid in making a clinical decision as to whether to continue adjuvant TMZ or start second-line therapy without delay. ${ }^{32}$

\section{Funding Statement}

This study was supported by a grant from the Korea Healthcare technology R\&D Projects, Ministry for Health, Welfare \& Family Affairs (HI13C0015) and by the Research Center Program of IBS (Institute for Basic Science) in Korea.

\section{References}

1. Brandes AA, Tosoni A, Spagnolli F, et al. Disease progression or pseudoprogression after concomitant radiochemotherapy treatment: pitfalls in neurooncology. Neuro Oncol 2008; 10:361-367.

2. Jeon HJ, Kong DS, Park KB, et al. Clinical outcome of concomitant chemoradiotherapy followed by adjuvant temozolomide therapy for glioblastaomas: single-center experience. Clin Neurol Neurosurg 2009; 111:679-682.

3. Wen PY, Kesari S. Malignant gliomas in adults. N Engl J Med 2008; 359:492-507.

4. Stupp R, Mason WP, van den Bent MJ, et al. Radiotherapy plus concomitant and adjuvant temozolomide for glioblastoma. N Engl J Med 2005; 352:987-996.

5. Brandsma D, Stalpers L, Taal W, Sminia P, van den Bent MJ. Clinical features, mechanisms, and management of pseudoprogression in malignant gliomas. Lancet Oncol 2008; 9:453-461.

6. Chaskis C, Neyns B, Michotte A, De Ridder M, Everaert H. Pseudoprogression after radiotherapy with concurrent temozolomide for high-grade glioma: clinical observations and working recommendations. Surg Neurol 2009; 72:423-428.

7. Taal W, Brandsma D, de Bruin HG, et al. Incidence of early pseudo-progression in a cohort of malignant glioma patients treated with chemoirradiation with temozolomide. Cancer 2008; 113:405-410.

8. Chamberlain MC, Glantz MJ, Chalmers L, Van Horn A, Sloan AE. Early necrosis following concurrent
Temodar and radiotherapy in patients with glioblastoma. J Neurooncol 2007; 82:81-83.

9. Young RJ, Gupta A, Shah AD, et al. MRI perfusion in determining pseudoprogression in patients with glioblastoma. Clin Imaging 2013; 37:41-49.

10. Chamberlain MC. Pseudoprogression in glioblastoma. J Clin Oncol 2008; 26:4359; author reply 4359-4360.

11. IchiharaE, KiuraK, Takigawa N, etal.Pseudoprogression of lung cancer after concomitant chemoradiotherapy. Jpn J Clin Oncol 2008; 38:140-142.

12. Gahramanov S, Raslan AM, Muldoon LL, et al. Potential for differentiation of pseudoprogression from true tumor progression with dynamic susceptibility-weighted contrast-enhanced magnetic resonance imaging using ferumoxytol vs. gadoteridol: a pilot study. Int J Radiat Oncol Biol Phys 2011; 79:514-523.

13. Brandes AA, Franceschi E, Tosoni A, et al. MGMT promoter methylation status can predict the incidence and outcome of pseudoprogression after concomitant radiochemotherapy in newly diagnosed glioblastoma patients. J Clin Oncol 2008; 26:2192-2197.

14. Mangla R, Singh G, Ziegelitz D, et al. Changes in relative cerebral blood volume 1 month after radiation-temozolomide therapy can help predict overall survival in patients with glioblastoma. Radiology 2010; 256:575-584.

15. van den Bent MJ, Vogelbaum MA, Wen PY, Macdonald DR, Chang SM. End point assessment in gliomas: novel treatments limit usefulness of classical Macdonald's Criteria. J Clin Oncol 2009; 27:2905-2908.

16. Abdel Razek AA, Kandeel AY, Soliman N, et al. Role of diffusion-weighted echo-planar MR imaging in differentiation of residual or recurrent head and neck tumors and posttreatment changes. AJNR Am J Neuroradiol 2007; 28:1146-1152.

17. Asao C, Korogi Y, Kitajima M, et al. Diffusionweighted imaging of radiation-induced brain injury for differentiation from tumor recurrence. AJNR Am J Neuroradiol 2005; 26:1455-1460.

18. Hein PA, Eskey CJ, Dunn JF, Hug EB. Diffusionweighted imaging in the follow-up of treated highgrade gliomas: tumor recurrence versus radiation injury. AJNR Am J Neuroradiol 2004; 25:201-209.

19. Lee WJ, Choi SH, Park CK, et al. Diffusion-weighted MR imaging for the differentiation of true progression from pseudoprogression following concomitant radiotherapy with temozolomide in patients with newly diagnosed high-grade gliomas. Acad Radiol 2012; 19:1353-1361.

20. Matsusue E, Fink JR, Rockhill JK, Ogawa T, Maravilla KR. Distinction between glioma progression and post-radiation change by combined physiologic MR imaging. Neuroradiology 2010; 52:297-306.

21. Zeng QS, Li CF, Liu H, Zhen JH, Feng DC. Distinction between recurrent glioma and radiation injury using magnetic resonance spectroscopy in combination with diffusion-weighted imaging. Int J Radiat Oncol Biol Phys 2007; 68:151-158.

22. Chu HH, Choi SH, Ryoo I, et al. Differentiation of true progression from pseudoprogression in glioblastoma 
treated with radiation therapy and concomitant temozolomide: comparison study of standard and highb-value diffusion-weighted imaging. Radiology 2013; 269:831-840.

23. Song YS, Choi SH, Park CK, et al. True progression versus pseudoprogression in the treatment of glioblastomas: a comparison study of normalized cerebral blood volume and apparent diffusion coefficient by histogram analysis. Korean J Radiol 2013; 14:662-672.

24. Kong DS, Kim ST, Kim EH, et al. Diagnostic dilemma of pseudoprogression in the treatment of newly diagnosed glioblastomas: the role of assessing relative cerebral blood flow volume and oxygen-6-methylguanine-DNA methyltransferase promoter methylation status. AJNR Am J Neuroradiol 2011; 32:382-387.

25. Wen PY, Macdonald DR, Reardon DA, et al. Updated response assessment criteria for high-grade gliomas: response assessment in neuro-oncology working group. J Clin Oncol 2010; 28:1963-1972.

26. Grossman RI, Hecht-Leavitt CM, Evans SM, et al. Experimental radiation injury: combined MR imaging and spectroscopy. Radiology 1988; 169:305-309.

27. Cha S, Knopp EA, Johnson G, Wetzel SG, Litt AW, Zagzag D. Intracranial mass lesions: dynamic contrast-enhanced susceptibility-weighted echo-planar perfusion MR imaging. Radiology 2002; 223:11-29.

28. Cha S, Lupo JM, Chen MH, et al. Differentiation of glioblastoma multiforme and single brain metastasis by peak height and percentage of signal intensity recovery derived from dynamic susceptibility-weighted contrast-enhanced perfusion MR imaging. AJNR Am J Neuroradiol 2007; 28:1078-1084.

29. Hoefnagels FW, Lagerwaard FJ, Sanchez E, et al. Radiological progression of cerebral metastases after radiosurgery: assessment of perfusion MRI for differentiating between necrosis and recurrence. J Neurol 2009; 256:878-887.

30. Baek HJ, Kim HS, Kim N, Choi YJ, Kim YJ. Percent change of perfusion skewness and kurtosis: a potential imaging biomarker for early treatment response in patients with newly diagnosed glioblastomas. Radiology 2012; 264:834-843.

31. Boxerman JL, Ellingson BM, Jeyapalan S, et al. Longitudinal DSC-MRI for distinguishing tumor recurrence from pseudoprogression in patients with a high-grade glioma. Am J Clin Oncol 2014. [Epub ahead of print]

32. Gahramanov S, Muldoon LL, Varallyay CG, et al. Pseudoprogression of glioblastoma after chemo- and radiation therapy: diagnosis by using dynamic susceptibility-weighted contrast-enhanced perfusion MR imaging with ferumoxytol versus gadoteridol and correlation with survival. Radiology 2013; 266:842-852.

33. Hu LS, Eschbacher JM, Heiserman JE, et al. Reevaluating the imaging definition of tumor progression: perfusion MRI quantifies recurrent glioblastoma tumor fraction, pseudoprogression, and radiation necrosis to predict survival. Neuro Oncol 2012; 14:919-930.
34. Tsien C, Galban CJ, Chenevert TL, et al. Parametric response map as an imaging biomarker to distinguish progression from pseudoprogression in high-grade glioma. J Clin Oncol 2010; 28:2293-2299.

35. Barajas RF, Chang JS, Sneed PK, Segal MR, McDermott MW, Cha S. Distinguishing recurrent intra-axial metastatic tumor from radiation necrosis following gamma knife radiosurgery using dynamic susceptibility-weighted contrast-enhanced perfusion MR imaging. AJNR Am J Neuroradiol 2009; 30:367-372.

36. Mangla R, Kolar B, Zhu T, Zhong J, Almast J, Ekholm S. Percentage signal recovery derived from MR dynamic susceptibility contrast imaging is useful to differentiate common enhancing malignant lesions of the brain. AJNR Am J Neuroradiol 2011; 32:1004-1010.

37. Hu LS, Baxter LC, Smith KA, et al. Relative cerebral blood volume values to differentiate high-grade glioma recurrence from posttreatment radiation effect: direct correlation between image-guided tissue histopathology and localized dynamic susceptibility-weighted contrast-enhanced perfusion MR imaging measurements. AJNR Am J Neuroradiol 2009; 30:552-558.

38. Sorensen AG. Perfusion MR imaging: moving forward. Radiology 2008; 249:416-417.

39. Varallyay CG, Muldoon LL, Gahramanov S, et al. Dynamic MRI using iron oxide nanoparticles to assess early vascular effects of antiangiogenic versus corticosteroid treatment in a glioma model. J Cereb Blood Flow Metab 2009; 29:853-860.

40. Haroon HA, Buckley DL, Patankar TA, et al. A comparison of Ktrans measurements obtained with conventional and first pass pharmacokinetic models in human gliomas. J Magn Reson Imaging 2004; 19:527-536.

41. Tofts PS, Brix G, Buckley DL, et al. Estimating kinetic parameters from dynamic contrast-enhanced $\mathrm{T}(1)$ weighted MRI of a diffusable tracer: standardized quantities and symbols. J Magn Reson Imaging 1999; 10:223-232.

42. Harrer JU, Parker GJ, Haroon HA, et al. Comparative study of methods for determining vascular permeability and blood volume in human gliomas. J Magn Reson Imaging 2004; 20:748-757.

43. Yun TJ, Park CK, Kim TM, et al. Glioblastoma treated with concurrent radiation therapy and temozolomide chemotherapy: differentiation of true progression from pseudoprogression with quantitative dynamic contrast-enhanced MR imaging. Radiology 2015; 274:830-840.

44. Cheng HL. Improved correlation to quantitative DCEMRI pharmacokinetic parameters using a modified initial area under the uptake curve (mIAUC) approach. J Magn Reson Imaging 2009; 30:864-872.

45. Liu G, Rugo HS, Wilding G, et al. Dynamic contrastenhanced magnetic resonance imaging as a pharmacodynamic measure of response after acute dosing of AG-013736, an oral angiogenesis inhibitor, in patients with advanced solid tumors: results from a phase I study. J Clin Oncol 2005; 23:5464-5473. 
46. Suh CH, Kim HS, Choi YJ, Kim N, Kim SJ. Prediction of pseudoprogression in patients with glioblastomas using the initial and final area under the curves ratio derived from dynamic contrast-enhanced T1-weighted perfusion MR imaging. AJNR Am J Neuroradiol 2013; 34:2278-2286.

47. Pope WB, Kim HJ, Huo J, et al. Recurrent glioblastoma multiforme: ADC histogram analysis predicts response to bevacizumab treatment. Radiology 2009; 252:182-189.

48. Noguchi T, Yoshiura T, Hiwatashi A, et al. Perfusion imaging of brain tumors using arterial spin-labeling: correlation with histopathologic vascular density. AJNR Am J Neuroradiol 2008; 29:688-693.

49. Schraml C, Müssig K, Martirosian P, et al. Autoimmune thyroid disease: arterial spin-labeling perfusion $\mathrm{MR}$ imaging. Radiology 2009; 253:435-442.

50. Cebeci H, Aydin O, Ozturk-Isik E, et al. Assesment of perfusion in glial tumors with arterial spin labeling; comparison with dynamic susceptibility contrast method. Eur J Radiol 2014; 83:1914-1919.

51. Jarnum H, Steffensen EG, Knutsson L, et al. Perfusion MRI of brain tumours: a comparative study of pseudocontinuous arterial spin labelling and dynamic susceptibility contrast imaging. Neuroradiology 2010; 52:307-317.

52. van Westen D, Petersen ET, Wirestam $\mathrm{R}$, et al. Correlation between arterial blood volume obtained by arterial spin labelling and cerebral blood volume in intracranial tumours. MAGMA 2011; 24:211-223.

53. Warmuth C, Gunther M, Zimmer C. Quantification of blood flow in brain tumors: comparison of arterial spin labeling and dynamic susceptibility-weighted contrast-enhanced MR imaging. Radiology 2003; 228:523-532.

54. Wolf RL, Wang J, Wang S, et al. Grading of CNS neoplasms using continuous arterial spin labeled perfusion MR imaging at 3 Tesla. J Magn Reson Imaging 2005; 22:475-482.

55. Choi YJ, Kim HS, Jahng GH, Kim SJ, Suh DC. Pseudoprogression in patients with glioblastoma: added value of arterial spin labeling to dynamic susceptibility contrast perfusion MR imaging. Acta Radiol 2013; 54:448-454.

56. Cha J, Kim ST, Kim HJ, et al. Differentiation of tumor progression from pseudoprogression in patients with posttreatment glioblastoma using multiparametric histogram analysis. AJNR Am J Neuroradiol 2014; 35:1309-1317.

57. Park JE, Kim HS, Goh MJ, Kim SJ, Kim JH. Pseudoprogression in patients with glioblastoma: assessment by using volume-weighted voxel-based multiparametric clustering of MR imaging data in an independent test set. Radiology 2015; 275:792-802. 